\title{
Strongly triggered collapse model confronts observations
}

\author{
Patrick Hennebelle ${ }^{1}$, Arnaud Belloche ${ }^{2}$, Philippe André ${ }^{3}$ \\ and Anthony Whitworth ${ }^{4}$ \\ ${ }^{1}$ Laboratoire de Radioastronomie Millimétrique, UMR 8112 du CNRS, École Normale \\ Supérieure et Observatoire de Paris, 24 rue Lhomond 75231 Paris Cedex 05 France \\ patrick.hennebelle@ens.fr \\ ${ }^{2}$ Max Planck-Institut fur Radioastronomie, Auf dem Hugel 69, 53121 Bonn, Germany \\ email: belloche@mpifr-bonn.mpg.de \\ ${ }^{3}$ Service d'Astrophysique, CEA/DSM/DAPNIA, C.E. Saclay, 91191 Gif-sur-Yvette Cedex, \\ France email: pandre@cea.fr \\ ${ }^{4}$ School of Physics \& Astronomy, Cardiff University, 5 The Parade, Cardiff CF24 3YB, Wales, \\ UK email: ant@astro.cf.ac.uk
}

\begin{abstract}
Detailed modelling of individual protostellar condensations is important to test the various theories. Here we present comparisons between strongly induced collapse models with one young class- 0 object, IRAS4A, in the Perseus cloud and one prestellar cloud observed in the Coalsack molecular cloud.
\end{abstract}

Keywords. stars:formation, hydrodynamics, gravitation

\section{Introduction}

Triggered star formation has been proposed since many years as an important mode of star formation. Indeed many sources of star formation triggering have been proposed and are discussed in this volume, namely turbulence, supernovae remnants, ionisation fronts, stellar outflows or large scale collapse. All of these processes ought to play a role in inducing star formation and probably have their own signatures. In order to be able to identify and to quantify their relative importance, it is necessary to study in great details protostellar condensations in which the collapse has been externally induced, looking for signatures of such violent triggering.

Here we present detailed comparisons between induced collapse models and 2 observed sources in which external triggering may have taken place, namely IRAS4A, a well observed class-0 condensation located in the NGC1333 complex, and the G2 globule located in the Coalsack molecular cloud.

In section 2, we first describe the models with which comparison will be performed. In section 3, comparison with IRAS4A is presented whereas comparison with the G2 globule is described in section 4 .

\section{Spontaneous versus induced collapse}

In order to compare in detail observations of protostellar cores with theoretical models, it is necessary to construct a class of models which on one hand is realistic enough but on the other hand sufficiently simple to be described by few parameters that can be easily varied. It is for example unlikely or at least computationally expensive to find in large scale turbulence simulations, a core which fits sufficiently well an observed object to allow very detailed comparisons. Our approach is as described in Hennebelle et al. $(2003,2004)$. 

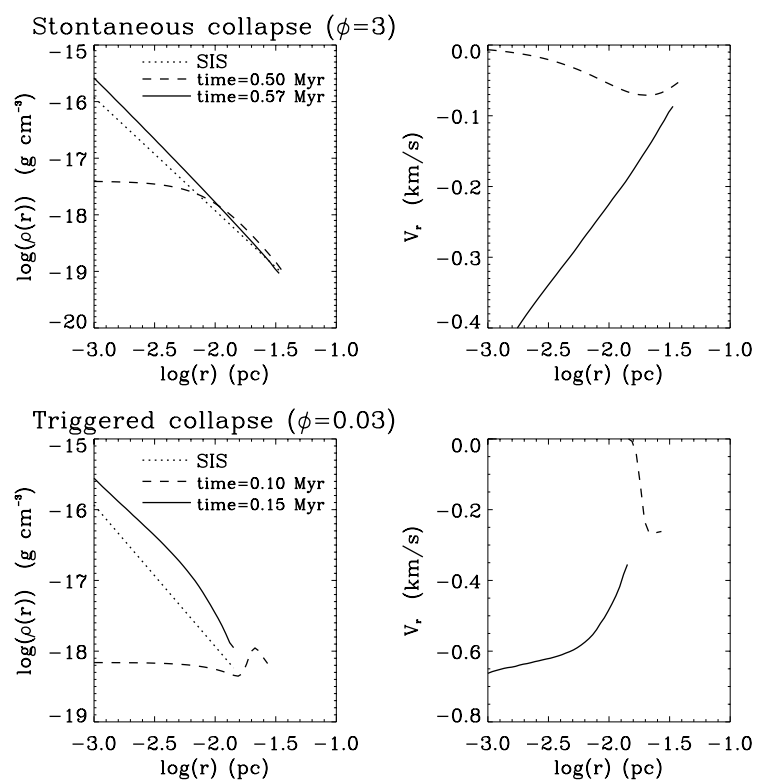

Figure 1. Density and velocity fields in the prestellar and class-0 phase for a spontaneous and a strongly triggered collapse.

We setup initially a stable Bonnor-Ebert sphere and we increase the external pressure at a given rate. In order to quantify the rapidity of the pressure increase, we define $\phi=\left(R_{c} / C_{s}\right) /(P / \dot{P})$ where $R_{c}$ is the cloud radius, $C_{s}$ the sound speed, $P$ the pressure and $\dot{P}$ its time derivative. $\phi$ represents therefore the ratio of the sound crossing time over the typical pressure increase time. Large values of $\phi$ correspond to slow pressure increase and describe a spontaneous collapse whereas for small values of $\phi$, the collapse is strongly externally triggered. The calculations have been done with the SPH technique.

Figure 1 shows the density and the velocity field in the equatorial plan before and after protostar formation for a spontaneous collapse $(\phi \simeq 3)$ and for a strongly triggered collapse $(\phi \simeq 0.03)$. In the first case, the density appears to be close to a Bonnor-Ebert sphere density in the prestellar phase, even when the cloud has become unstable, and close to the density of the singular isothermal sphere (SIS) during the class-0 phase. The velocity remains subsonic during the prestellar phase and in the core outer part in the class-0 phase. It becomes more and more supersonic as the collapse proceeds, in the inner part. The situation is much different for $\phi=0.03$. The velocity is supersonic in the outer part of the core during prestellar phase and everywhere during class- 0 phase. The density is also very different from the previous case. It is higher in outer part than in the center. Indeed the strong pressure increase has launched a compression wave that propagates inwards. During the class-0 phase, the density is significantly denser than the SIS density. All of these features appear therefore to be characteristic of externally induced collapse.

\section{Comparison with the young class-0 IRAS4A}

NGC1333 is a well studied region of the Perseus cloud. Several outflows have been detected (Knee \& Sandell 2000), at least one of them is pointing towards IRAS4A making the possibility of induced collapse for this source rather plausible. IRAS4A has been observed in various molecular lines by Di Francesco et al. (2001) and in the contin- 


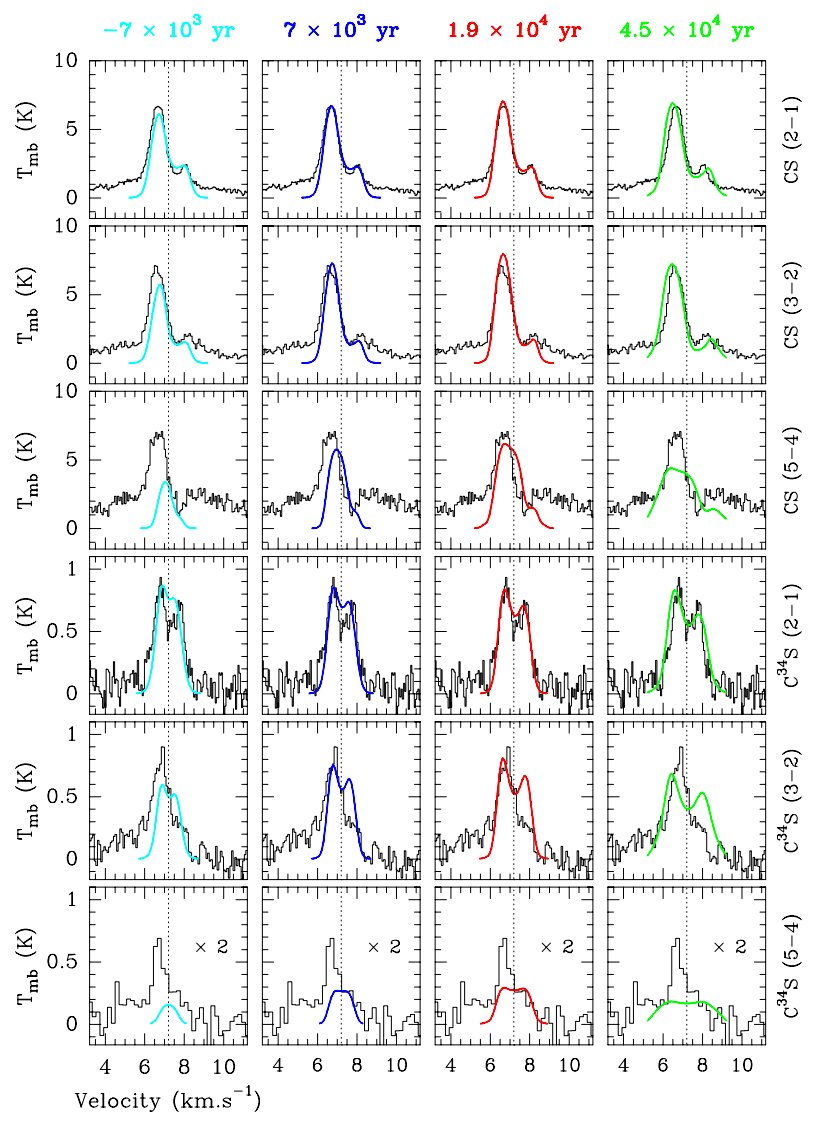

Figure 2. Comparison between observed spectra and synthetic spectra obtained from the strongly induced collapse at various time.

uum by Motte \& André (2001). Di Francesco et al. (2001) inferred supersonic velocity (0.5-1 km/s) whereas Motte \& André (2001) observed density up to 10 times the SIS density. As recalled in previous section, both are signatures of induced collapse. This has been confirmed by observations done recently by Belloche et al. (2006).

In order to confirm the scenario of induced collapse for IRAS4A and to set accurate constraints, we have calculated synthetic spectra in the available lines, using the radiative transfer code described in Belloche et al. (2002) for the model $\phi=0.03$ presented in the previous section. Figure 2 shows the comparison between the data and the synthetic profiles at 4 time steps before and after protostellar formation. The comparison reveals that good agreement can be obtained for almost all lines at time $1.9 \times 10^{4} \mathrm{yr}$ whereas at earlier times synthetic lines are generally too narrow and at later times, they are generally too broad. As can be seen various features are not well reproduced even at time $1.9 \times 10^{4} \mathrm{yr}$. For example the large wings seen in $\mathrm{CS}(2-1)$ are most probably due to the outflow launched by IRAS4A which is not taken into account in our modelling. It is also seen that $\mathrm{C}^{34} S(5-4)$ is poorly reproduced. We speculate that the reason for this disagreement is that this line being optically thin, it traces the outer part of the core. The asymmetry of the lines may reveal that the collapse on large scales is indeed not symmetric.

We conclude that IRAS4A can be reasonably well reproduced by our model although further refinements are highly wishable. 

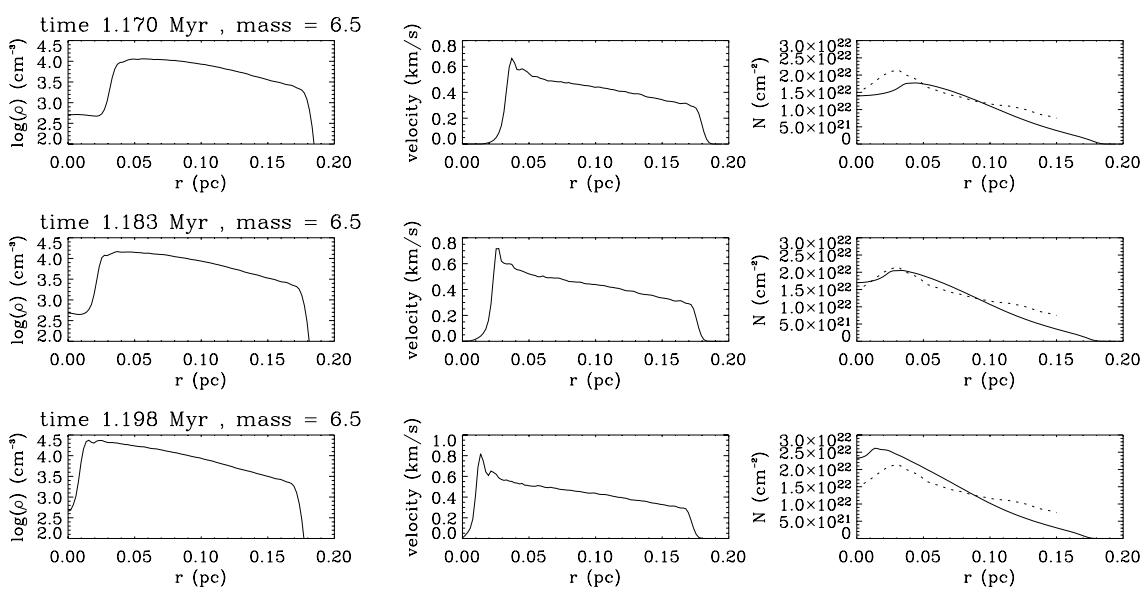

Figure 3. Density, velocity and column density of the collapsing prestellar cloud (solid lines) and column density inferred from observations (dotted line) of Lada et al. (2004).

\section{Comparison with the prestellar G2 globule of Coalsack}

Lada et al. (2004) have recently observed in the Coalsack molecular cloud a core which presents an unusual ring-like structure around its centre. The column density appears therefore to decrease inwards when one approaches the core centre. Qualitatively this feature may have similarity with the compression wave shown in figure 1 . In our model the compression wave is a dense shell which in projection could be seen as a ring. To test the validity of this scenario, we have compared the column density observed by Lada et al. (2004) with the column density of our model at various time steps (Hennebelle et al. 2006). Figure 3 displays the density, velocity and column density fields at 3 time steps as well as the column density observed by Lada et al. (2004). The column density of the second case appears to be in good agreement with these observations. However no conclusion should be drawn until kinematical data are available for this core (only one spectrum has been observed). Indeed we have calculated synthetic spectra, under optically thin assumption, towards various positions. Our model predicts that toward the centre, the lines should be strongly split. This prediction should be easy to test and we look forward to future observations of this peculiar object.

\section{References}

Belloche, A., André P., Despois, D. \& Blinder S., 2002, A\&̛A 393, 927

Belloche, A., Hennebelle, P. \& André P., 2006, A\& A 453, 145

Di Francesco, J., Myers, P., Wilner, D., Ohashi, N. \& Mardones, D., 2001, ApJ 562, 770

Hennebelle, P., Whitworth, A., Gladwin, P. \& André, P., 2003, MNRAS 340, 870

Hennebelle, P., Whitworth, A., Cha, S.-H. \& Goodwin, S. P., 2004, MNRAS 348, 687

Hennebelle, P., Whitworth, A. \& Goodwin, S., 2006, A\&A 451, 141

Knee, L. \& Sandell, G., 2000, A\&A 361, 671

Lada, C., Huard, T., Crews, L. \& Alves, J., 2004, ApJ 610, 303

Motte, F. \& André, P., 2001, A\&A 365, 440

\section{Discussion}

BARsony: (1.) Regarding your modeling of NGC 1333 IRAS The initial conditions you use require a very fast timescale for the external pressure change which triggered the collapse, in order to match the density distribution of the infalling envelope on scales of 
$1500<r<10000$ AU from millimeter continuum observations (Belloche et al. 2006). Furthermore, you argue that such a fast external compression is necessary to account for the observed high accretion rate of $10^{-4} \mathrm{M}_{\odot} \mathrm{yr}^{-1}$. Does this mean that you would expect ALL Class 0 sources to have been triggered (since their accretion rates are so high)? (2.) In your model, you cannot match the density structure of the protostellar envelope at scales of a few $\times 100$ AU $<r<1500$ AU. Why?

Hennebelle: (1.) It depends on whether or not the infall is observed to be supersonic or subsonic. (2.) The model does not take rotation of magnetic fields into account. Including these may lead to better agreement with observations. 